\title{
Case presentation commentary on "ammonia for positron emission tomography (PET): A promising technique for patients with LBBB and suspicion of coronary artery disease"
}

\author{
Lawrence $M$. Phillips, $M D^{a}$ \\ a Division of Cardiology, NYU Langone Medical Center, New York
}

Received Jun 10, 2018; accepted Jun 11, 2018

doi: $10.1007 / \mathrm{s} 12350-018-1345-1$

In this issue of the Journal of Nuclear Cardiology, Bafadel et al. present a case of multimodality approaches to evaluation of ischemia in a patient with a baseline LBBB on EKG. ${ }^{1}$ This patient was found to have at least moderate left anterior descending artery stenosis based on CTA and subsequently underwent both SPECT and PET exercise testing for evaluation of functional status and evidence of ischemia in this anatomic distribution.

A key teaching point in this case is the impact that a LBBB on EKG can have on the ability of the nuclear reader to interpret perfusion abnormalities. For this reason, the American Society of Nuclear Cardiology Imaging Guidelines recommend that patients with LBBB should preferentially undergo pharmacologic vasodilator stress tests when having an imaging stress test. $^{2}$ It is further clarified that the patients should not use dobutamine as a pharmacologic agent due to its ability to increase the heart rate similarly to exercise.

In this case, the 12NH3-PET images showed no reversible perfusion defects in the septum compared to the perfusion defect seen in the post-perfusion images in the SPECT test. One concern is the decreased exercise time in the PET exam (7 minutes) compared to the
SPECT exam (10 minutes). It can be hypothesized that the peak heart rate in the PET exam was lower which might also account for the lack of septal artifact.

This case presents a potential change in diagnostic approach to patients undergo functional assessment with a baseline LBBB. As noted in the paper, further comparative studies will be needed before a change should take place in the recommended protocols.

\section{Disclosure}

Dr. Phillips declares that there is no conflict of interest to disclose.

\section{References}

1. Bafadel A, Meredith R, Xu B, Jaber W. Ammonia for exercise positron emission tomography (PET): a promising technique for patients with LBBB and suspicion of coronary artery disease. A case study. J Nucl Cardiol 2018. https://doi.org/10.1007/s12350018-1320-x.

2. Henzlova MJ, Duvall WL, Einstein AJ, Travin MI, Verberne HJ. ASNC imaging guidelines for SPECT nuclear cardiology procedures: stress, protocols, and tracers. J Nucl Cardiol 2016;23:606-39.
Reprint requests: Lawrence M. Phillips, MD, Division of Cardiology, NYU Langone Medical Center, New York; lawrence.phillips@nyumc.org

J Nucl Cardiol 2018;25:1089.

$1071-3581 / \$ 34.00$

Copyright (c) 2018 American Society of Nuclear Cardiology. 